\title{
Pupil Detection and Tracking Based on a Round Shape Criterion by Image Processing Techniques for a Human Eye-Computer Interaction System
}

\author{
Tsumoru Ochiai and Yoshihiro Mitani
}

\begin{abstract}
The pupil detection and tracking is one of important steps for developing a human-computer interaction system. To develop a human eye-computer interaction system, we study pupil detection and tracking by image processing techniques. In the image processing, the illumination directly influences the image quality in general. If influence of illumination is little, we can obtain an image of good image quality. Therefore, we proposed a hardware constitution of an infrared light-emitting diode (LED) light, a sensitive infrared camera, and an infrared (IR) filter for pupil detection and tracking, in order to avoid the influence of illumination. However, detecting a pupil was still poor because only thresholding was carried out in a pupil image. The previously proposed method has no consideration of a round shape criterion to detect a pupil part. In this paper, in order to detect a pupil more accurately, we have proposed to use a round shape criterion of a pupil. In the experiment, we investigate the robustness of pupil detection and tracking by image processing techniques for a human eye-computer interaction system.
\end{abstract}

Index Terms-Human-computer interaction, image processing techniques, pupil detection and tracking, round shape criterion, infrared filter.

\section{INTRODUCTION}

In an environmental control system, users always wear some devices. The devices are said to be a contact type. However, if a user uses a contact type device for a long time, the user must work hard to endure pain. The contactless type device is expected to relieve a user's pain. By the development of a human-computer interaction system [1], [2], various contactless type devices have been proposed [3], [4]. A device using a human eye is one of contactless type devices [4]. In a human eye-computer interaction system, we need to understand eye movement to detect an eye. In this paper, to detect an eye accurately, we focus on a pupil of an eye. The pupil detection and tracking is one of important steps for developing a human-computer interaction system [4]. To develop a human eye-computer interaction system, we study pupil detection and tracking by image processing techniques [5], [6]. In the image processing techniques, the illumination directly influences the image quality in general [7]. If influence of the illumination is little, we can obtain an image of good image quality. The subsequent image processing techniques are expected almost to succeed.

By a specific device using active infrared (IR) illumination,

Manuscript received March 29, 2016; revised on May 30, 2016.

Ochiai Tsumoru and Yoshihiro Mitani are with the National Institute of Technology, Ube College, Ube, Japan (e-mail: mitani@ ube-k.ac.jp). an effective eye tracking method is proposed [8]. The specific device can get 2 types of images: dark and bright images. We want to use not a specific device but a more general device. Therefore, we proposed a hardware constitution of an infrared light-emitting diode (LED) light, a sensitive infrared camera, and an infrared (IR) filter for pupil detection and tracking, in order to avoid the influence of illumination [9]. From the experimental results, the combination of an infrared LED light, a sensitive infrared camera, and an IR filter yields a favorable performance. However, detecting a pupil was still poor because only thresholding was carried out in a pupil image. The previously proposed method [9] has no consideration of a round shape criterion to detect a pupil part.

In this paper, in order to detect a pupil more accurately, we have proposed to use a round shape criterion of a pupil part. In the experiment, we investigate the robustness of pupil detection and tracking by image processing techniques for a human eye-computer interaction system.

In Section II, a previously proposed method is described [9]. The hardware constitution and pupil detection are shown. Section III shows the proposed method. To detect a pupil part more accurately, we propose to use a round shape criterion of a pupil. Section IV and Section V show the experiment and conclusions.

\section{A PREvious Work}

In a previous study [9], we have proposed a human eye-computer interaction system. The hardware constitution and pupil detection are described.

\section{A. Hardware Constitution}

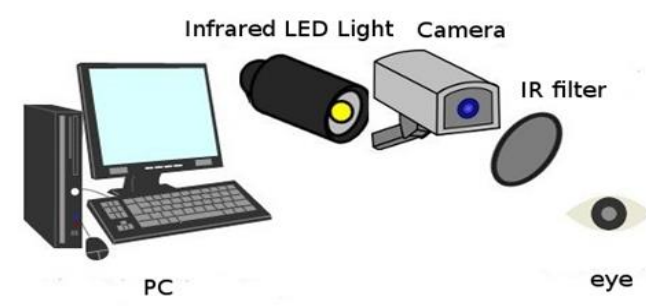

Fig. 1. A human eye-computer interaction system.

Fig. 1 shows the hardware constitution of a human eye-computer interaction system. The hardware consists of a personal computer, an infrared LED light, a sensitive infrared camera, and an IR filter. A human eye is recorded as a video through a camera with an IR filter. From this recorded video, the method of pupil detection, pupil center calculation, and pupil tracking, is carried out by image processing techniques. 
In order to accurately detect and track a pupil of an eye by image processing techniques, we want to reduce the illumination influence as much as possible. It is known that a pupil has a characteristic which does not reflect an infrared light. Thus, we focus on this characteristic and consider using an infrared light. In general, in the case of the use of a digital camera oriented for a visible light such as a web camera, these cameras may cut an infrared light. This leads to difficulty that we cannot use reflection of an infrared light. We use a sensitive infrared camera. The images obtained by a sensitive infrared camera still include many noises. Therefore, we use an infrared (IR) filter and expected to reduce the influence of noises. In the experiments, we use an IR filter which cuts the wavelength of light under $800 \mathrm{~nm}$. With the IR filter, the camera is taken into an infrared light only. It effects to reduce influences of the illumination or noises. A part of an eye except a pupil reflects an infrared light. On the other hand, a pupil does not reflect an infrared light at all. Thus, a pupil in the video is comparatively easily detected. By an image processing technique: thresholding, a pupil part is detected. That is, by setting a comparatively small threshold value for thresholding, we can detect the pupil part.

\section{B. Pupil Detection}

We show pupil detection by image processing techniques for a human eye-computer interaction system. The initial image in a video is transformed into a gray scale image. The gray scale level is 256 . By thresholding with an appropriate threshold value, only a pupil part can be detected. The threshold value can be given by a user while watching a video dynamically. A pupil center is calculated from an intensity frequency distribution. The pupil center is regarded as a center of gravity of its distribution. The center of gravity of the pupil part is calculated as follows:

$$
\begin{gathered}
\left(\mathrm{x}_{G}, y_{G}\right)=\left(\frac{\mu_{1,0}}{\mu_{0,0}}, \frac{\mu_{0,1}}{\mu_{0,0}}\right) \\
\mu_{m, n}=\sum_{X} \sum_{y} x^{m} y^{n} f(x, y)
\end{gathered}
$$

Here, the $\mu_{m, n}$ denotes the m- and n- order image moment. The $\mu_{0,0}$ is an area of a pupil part.

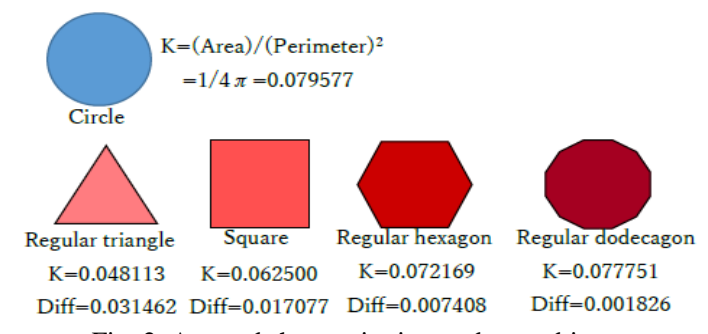

Fig. 2. A round shape criterion to detect objects.

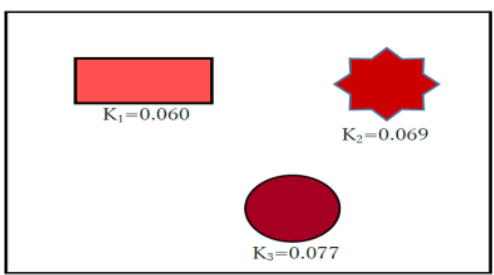

Fig. 3. An example of pupil recognition based on a round shape criterion.

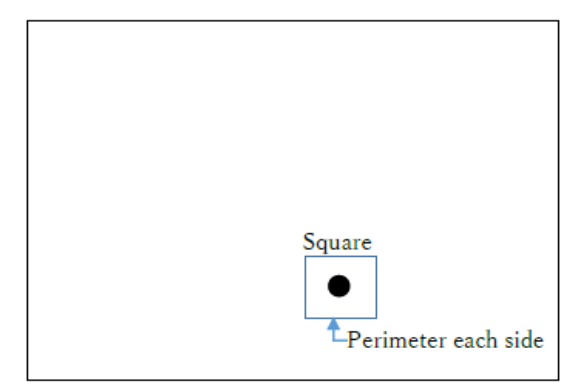

Fig. 4. A tracking assignment area of a pupil part.

\section{A PROPOSED METHOD}

In order to detect a pupil more accurately, we have proposed to use a round shape criterion of a pupil part. A proposed method is based on the previously proposed method [9]. The hardware constitution is the same. In pupil detection after thresholding a pupil part, the proposed method differs. We add a round shape criterion which may lead to robustness of detecting a pupil. Once a pupil part is successfully detected, by an assignment area of a pupil part, tracking a pupil is carried out only in the assignment area. The cost to compute image processing is expected to be down. The direction of an eye to move from a frame to a next frame is also shown. We describe each of a round shape criterion, a tracking assignment area of a pupil part, and a direction of an eye movement.

\section{A. Round Shape Criterion}

By adding a round shape criterion after thresholding a pupil part, a robust detection of a pupil is expected. We define a round shape criterion as follows:

\section{$K=($ Area $) /($ Perimeter $) 2$}

Here, Area and Perimeter are area and perimeter of an object to be detected by thresholding, respectively. If the number of pixels in an object is 150 and under or 10,000 and over, the objects are regarded as noises and removed.

Fig. 2 shows a round shape criterion to detect objects. A pupil part is considered to close a circle. We regard a pupil part as a circle. A value of $K$ with a circle is calculated as $1 /(4 \pi)=0.079577$. Note that the $K=1 /(4 \pi)$ is independent of the radius. If a pupil part is detected, the value of $K$ is near $1 /(4 \pi)$. In Fig. $2, K$ s values of regular triangle, square, regular hexagon, and regular dodecagon are 0.048113, 0.062500, 0.072169 , and 0.077751 , respectively. The differences of each of objects and a circle in terms of $K$ are 0.031462 , $0.017077,0.007408$, and 0.001826 , respectively. As the $K$ s values get grow, the differences get smaller. The shape goes a circle more and more

Fig. 3 shows an example of pupil recognition based on a round shape criterion. In fig. 3 , three objects are detected and their $K s$ values are calculated. The values of $K 1, K 2$, and $K 3$ are $0.060,0.069$, and 0.077 , respectively. Among $K s$ values, $K 3(=0.077)$ is the nearest to $1 /(4 \pi)=0.079577$. Therefore, an object with $K 3$ is recognized as a pupil. Others are removed.

\section{B. Tracking Assignment Area of a Pupil Part}

We show a tracking assignment area of a pupil part. Every frame, a pupil detection is performed. However, the cost to 
compute a pupil detection is high. Therefore, we have proposed to use a tracking assignment area of a pupil part. Once a pupil is successfully detected, by an assigning area of a pupil part, tracking a pupil is carried out only in the assignment area. The effects lead to a cost reduction. If an object within a tracking assignment area meets the following equation, a pupil detection is performed only in the assignment area.

$$
1 /(4 \pi) \text {-(Area) } /(\text { Perimeter }) 2<0.03
$$

In the equation, a value 0.03 is determined by a preliminary experiment. Fig. 4 shows a tracking assignment area of a pupil part. The area is a square with a perimeter every side. If the equation doesn't meet, we re-compute, i.e., firstly thresholding the image into pupil part candidates. Secondly with a round shape criterion, a pupil is detected. While focusing on a tracking assignment area of a pupil part, outside of the area is ignored. If there is something like an eye outside the area, the object is never detected. By the use of a tracking assignment area of a pupil part, the computational cost leads to reduction.

\section{Direction of an Eye Movement}

We show a direction of an eye movement. The eye movement is classified into 9 conditions, 8 directions, left, right, upper, lower, left-upper, right-upper, left-lower, and right-lower, and stay. The center of the first square in a frame is located on the gravity center of a pupil area. The square side is 15 pixels. If the gravity center of a pupil area in a next frame is over the square, we classified an eye movement into 8 directions. Otherwise, the gravity center of a pupil area still remains within the square. The eye movement stays.

\section{EXPERIMENTAL RESULTS}

The proposed method is compared with a previously proposed method [9], in terms of detecting a pupil part, not objectively but subjectively. From a number of experimental results compared to the previous method [9], the proposed method has shown to be more effective to detect a pupil part stably and accurately. A speed of tracking a pupil seems more quickly. The positive effect of adding a round shape criterion shows clearly.

Fig. 5 shows an example of the proposed method to detect a pupil part stably. The left and right images show a real movie with a result and an image processing by the proposed method, respectively. A plus sign denotes the gravity of a pupil part to be detected. The square is located on the center of the gravity, and its side is a perimeter of a pupil. The value 0.070289 is a value of $\mathrm{K}$. The value is near $1 /(4 \pi)=0.079577$. In the right image, there are many black areas by thresholding. The right sided black areas are considered to be noises. We want to exclude a right sided large piece. By the proposed method, we can get a pupil part accurately. The other black areas are not detected.

Fig. 6 shows an example of the proposed method to track an eye movement. In the image, a pupil part is successfully detected by the proposed method. The left side of the image shows a direction of an eye movement. The 9 conditions are shown from a frame to a next frame. Note that the 9 conditions are the 8 directions and a stay status. This seems good largely, because a comparatively quick response appears with an eye movement.

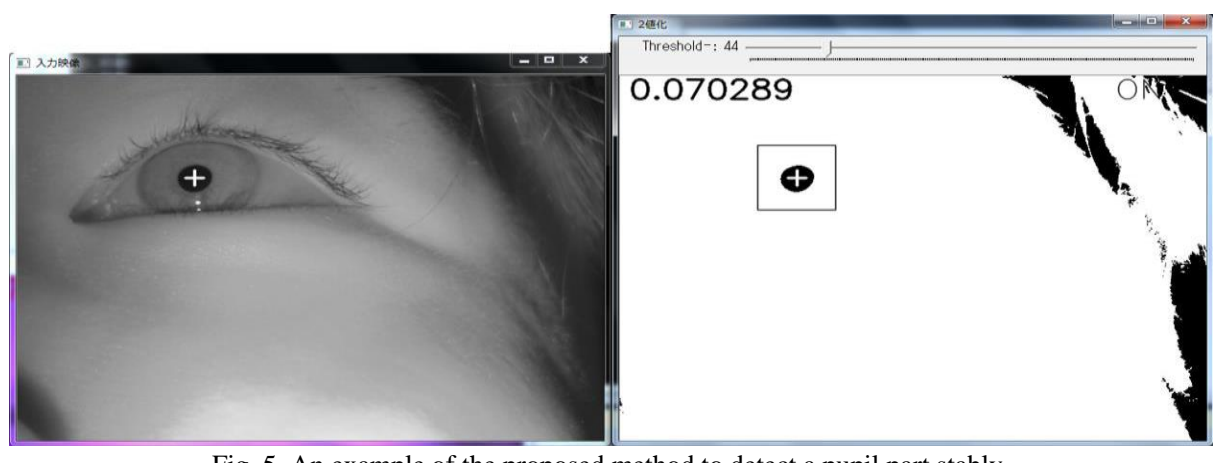

Fig. 5. An example of the proposed method to detect a pupil part stably.

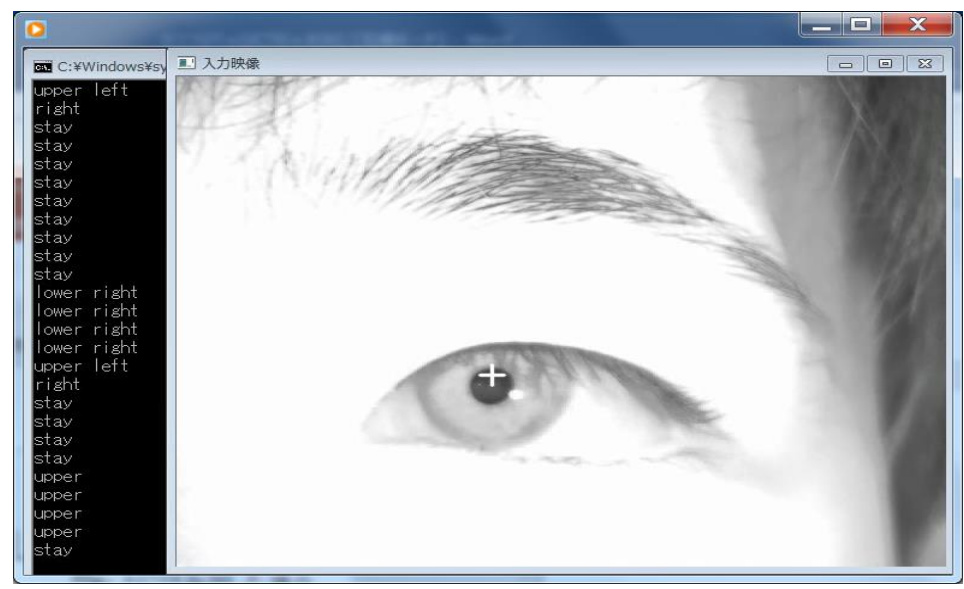

Fig. 6. An example of the proposed method to track an eye movement. 


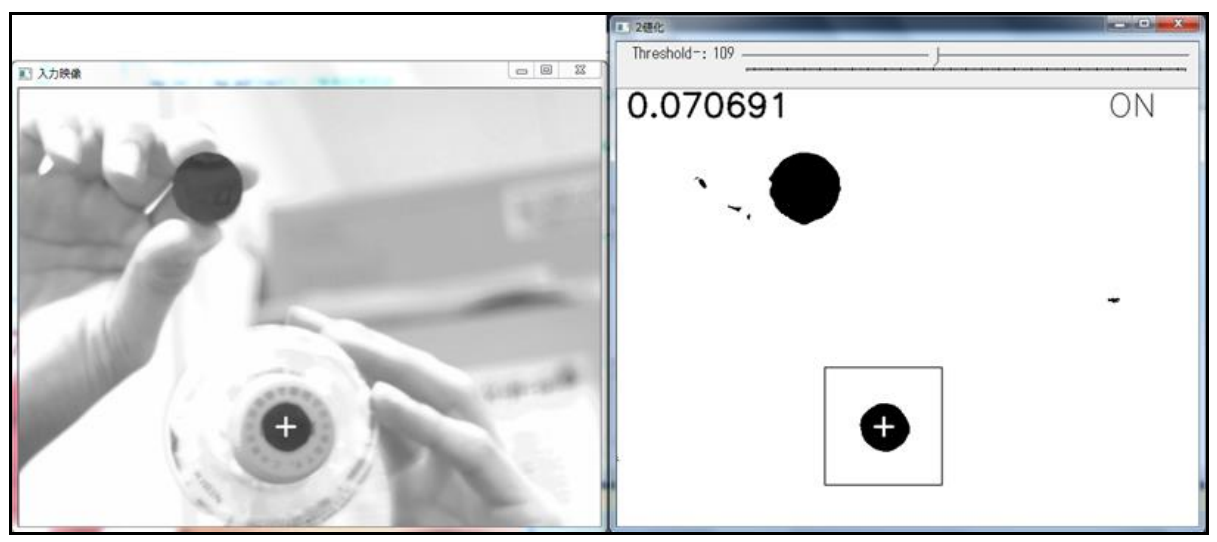

Fig. 7. An example of the proposed method for a tracking assignment area of a pupil part.

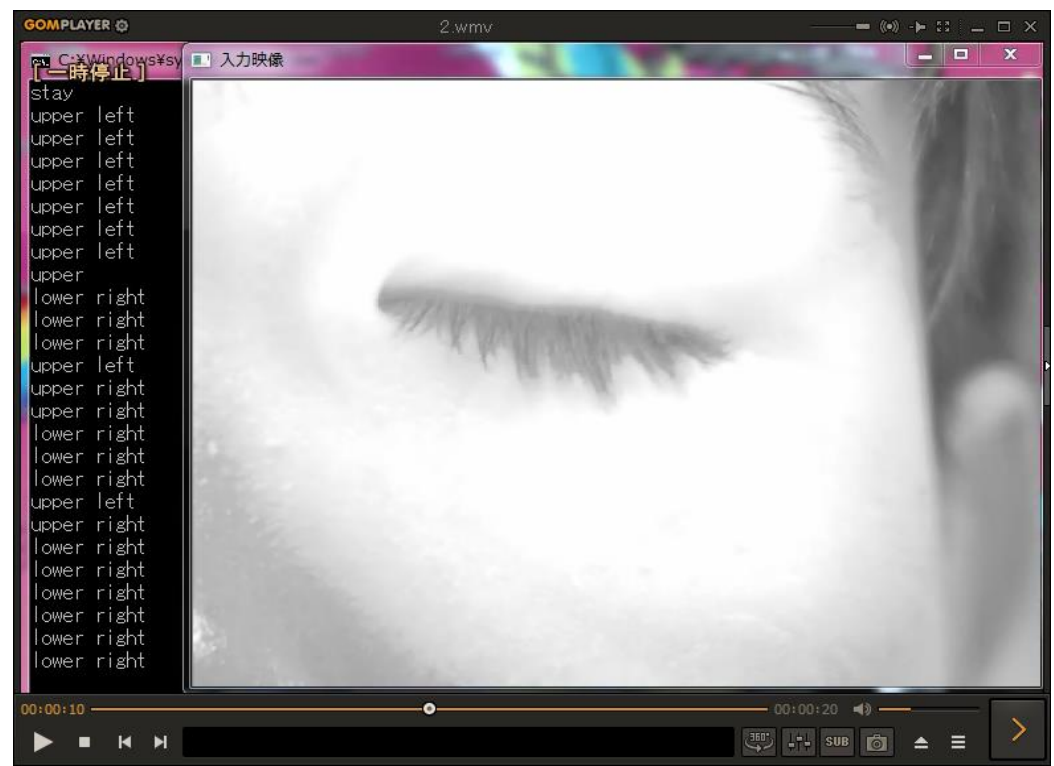

Fig. 8. A failure example of the proposed method for a blink of an eye.

Fig. 7 shows an example of the proposed method for a tracking assignment area of a pupil part. For reduction of a computational cost, once we get a pupil part, we focus not on the whole image but a tracking assignment area of a pupil part. In the experiments, we use 2 hand-made eyes. Once an eye is detected, the processing area is limited. Even if an object like an eye appears outside of a tracking assignment area of a pupil part, the object is not detected. In our limited experiments, we confirm that a tracking assignment area works well.

\section{CONCLUSIONS}

In this paper, in order to detect a pupil more accurately, we have proposed to use a round shape criterion of a pupil part. In the experiment, we investigate the robustness of pupil detection and tracking by image processing techniques for a human eye-computer interaction system. From the experimental results, the proposed method outperforms a previously proposed method [9] to detect and track a pupil part accurately, stably, and quickly. We believe the proposed method is more robust.

However, the proposed method still remains some problems. A very quick move of an eye is not good at detecting a pupil. A blink of an eye is not yet supported. Fig. 8 shows a failure example of the proposed method for a blink of an eye. If a pupil is successfully detected, the plus sign of a center of a pupil usually appears. In the image of fig. 8, the plus sign of the gravity of a pupil disappears. Furthermore, we use an infrared light in the hardware constitution. Therefore, by using a visible light, we also explore a human eye-computer interaction system. Recently, pupil detection methods are evaluated [10]. In the future, we investigate methods described in the paper [10].

\section{ACKNOWLEDGMENT}

We wish to thank students, Mr. Miyagi in Kyushu Institute of Technology and Mr. Shimata in National Institute of Technology, Ube College. We also thank referees for careful reading our manuscript and for giving useful comments, especially for suggesting the better terms and sentences.

\section{REFERENCES}

[1] I. S. MacKenzie, "Fitts' law as a research and design tool in human-computer interaction," Human-Computer Interaction, vol. 7, no.1, pp. 91-139, 1992.

[2] A. Jaimes and N. Seba, "Multimodal human-computer interaction: A survey," Computer Vision and Image Understanding, vol. 108, no. 1-2, pp. 116-134, 2007.

[3] V. I. Pavlovic, R. Sharma, and T. S. Huang, "Visual interpretation of hand gestures for human-computer interaction: A review," IEEE PAMI, vol. 19 , no. 7 , pp. $677-695,1997$.

[4] D. W. Hansen and Q. Ji, "In the eye of the beholder: a survey of models for eyes and gaze," IEEE PAMI, vol. 32, no. 3, pp. 478-500, 2010. 
[5] J. C. Russ, The Image Processing Handbook, 3rd ed., CRC Press, 1999.

[6] W. K. Pratt, Digital Image Processing, 3rd ed., A Wiley-Interscience Publication, 2001.

[7] S. Z. Li, R. Chu, S. Liao, and L. Zhang, "Illumination invariant face recognition using near-infrared images," IEEE PAMI, vol. 29, no. 4, pp. 627-639, 2007.

[8] Z. Zhiwei, J. Qiang, K. Fujimura, and L. Kuangchih, "Combining Kalman filtering and mean shift for real time eye tracking under active IR illumination," in Proc. 16th Intl. Conf. on Pattern Recognition, 2002 pp. 318-321.

[9] R. Shimata, Y. Mitani, and T. Ochiai, "A study of pupil detection and tracking by image processing techniques for a human eye-computer interaction system," in Proc. 16th IEEE/ACIS International Conference on Software Engineering, Artificial Intelligence, Networking and Parallel/Distributed Computing, 2015, pp. 545-548.

[10] W. Fuhl, M. Tonsen, A. Bulling, and E. Kasneci, "Pupil detection for head-mounted eye tracking in the wild: An evaluation of the state of the art," Machine Vision and Applications, vol. 27, no. 8, pp. 1275-1288.

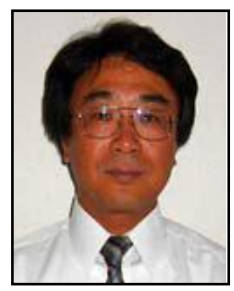

Tsumoru Ochiai got the Ph.D degree and is currently a professor at National Institute of Technology, Ube College, Japan. His research interests include mechatronics and image processing techniques.

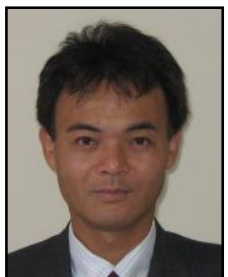

Yoshihiro Mitani got the Ph.D degree and is currently a professor at National Institute of Technology, Ube College, Japan. His research interests include pattern recognition and image processing techniques. He is a member of IEEE. 